\title{
Multiplicity Results of Positive Radial Solutions for $p$-Laplacian Problems in Exterior Domains
}

\author{
Chan-Gyun Kim, Yong-Hoon Lee, and Inbo Sim \\ Department of Mathematics, Pusan National University, Pusan 609-735, South Korea
}

Correspondence should be addressed to Yong-Hoon Lee, yhlee@pusan.ac.kr

Received 23 December 2007; Accepted 16 March 2008

Recommended by Zhitao Zhang

We find the second positive radial solution for the following $p$-Laplacian problem: $\operatorname{div}\left(|\nabla u|^{p-2} \nabla u\right)+$ $K(|x|) u^{q}=0$ in $\Omega,\left.u\right|_{\partial \Omega}=0, u(x) \rightarrow \mu>0$ as $|x| \rightarrow \infty$, where $\Omega=\left\{x \in \mathbb{R}^{N}:|x|>r_{0}\right\}, r_{0}>0$, $N>p>1, K \in C(\Omega,(0, \infty))$ and $q>p-1$. We also give some global existence results with respect to the parameter $\mu$.

Copyright $(92008$ Chan-Gyun Kim et al. This is an open access article distributed under the Creative Commons Attribution License, which permits unrestricted use, distribution, and reproduction in any medium, provided the original work is properly cited.

\section{Introduction}

In this paper, we study the existence, nonexistence, and multiplicity of positive radial solutions for the following $p$-Laplacian problem:

$$
\begin{aligned}
& \operatorname{div}\left(|\nabla u|^{p-2} \nabla u\right)+K(|x|) u^{q}=0 \text { in } \Omega, \\
& \left.u\right|_{\partial \Omega}=0, \quad u \longrightarrow \mu>0 \quad \text { as }|x| \longrightarrow \infty, \\
& \left.u\right|_{\partial \Omega}=\mu>0, \quad u \longrightarrow 0 \text { as }|x| \longrightarrow \infty,
\end{aligned}
$$

where $\Omega=\left\{x \in \mathbb{R}^{N}:|x|>r_{0}>0\right\}, N>p>1, \mu$ is a positive real parameter, $K \in C\left(\left(r_{0}, \infty\right), \mathbb{R}^{+}\right)$ with $\mathbb{R}^{+}=(0, \infty)$.

The present work is motivated by Deng and Li [1] who consider a semilinear problem of the form

$$
\begin{gathered}
\Delta u+K(x) u^{q}=0 \text { in } \Omega, \\
u>0 \text { in } \Omega, \quad u \in H_{\mathrm{loc}}^{1}(\Omega) \cap C(\bar{\Omega}), \\
\left.u\right|_{\partial \Omega}=0, \quad u \longrightarrow \mu>0 \text { as }|x| \longrightarrow \infty,
\end{gathered}
$$


where $\Omega=\mathbb{R}^{N} \backslash \omega$ is an exterior domain in $\mathbb{R}^{N}, \omega \subset \mathbb{R}^{N}$ is a bounded domain with smooth boundary, and $N>2, q>1$. Among other results, they prove under the assumption that

$\left(K_{1}\right) K \in C_{\text {loc }}^{\alpha}(\Omega), K \geq 0, K \not \equiv 0$, and there exist $C, \epsilon, M>0$ such that $|K(x)| \leq C|x|^{-l}$ for $|x| \geq M$ with $l \geq 2+\epsilon$

that there exists $\mu^{*}>0$ such that $(D L)$ has at least one solution for $\mu \in\left(0, \mu^{*}\right)$ and no solution for $\mu \in\left(\mu^{*}, \infty\right)$. Furthermore, if $K \in L^{1}(\Omega)$, then the solution at $\mu=\mu^{*}$ exists and is unique.

We expect that problem $(D L)$ may have certain bifurcation phenomenon of solutions with respect to $\mu$ so that there should be at least one more solution for $\mu \in\left(0, \mu^{*}\right)$. This is our goal for this paper and our first result comes out as follows. Assume $q>p-1$ and

$(K)$ there exists $\beta>p-1$ such that $\int_{r_{0}}^{\infty} r^{\beta} K(r) d r<\infty$.

Then, there exist $\mu_{0} \geq \mu^{*}>0$ such that $(P)+\left(D_{i}\right), i=1,2$, has at least two positive radial solutions for $\mu \in\left(0, \mu^{*}\right)$, at least one positive radial solution for $\mu \in\left[\mu^{*}, \mu_{0}\right]$ and no positive radial solution for $\mu \in\left(\mu_{0}, \infty\right)$.

We notice that this result is partial since the existence of multiple solutions on interval $\left[\mu^{*}, \mu_{0}\right]$ is not obvious. This is mainly caused by coarse topological structure of solution space. If indefinite weight $K(|x|)$ is of the form $|x|^{-l}$ with $l>p$, then we can prove $\mu^{*}=\mu_{0}$ in the above conclusion for problem $(P)+\left(D_{1}\right)$, that is, there exists $\mu^{*}>0$ such that $(P)+\left(D_{1}\right)$ has at least two, one, or no positive solutions according to $\mu \in\left(0, \mu^{*}\right), \mu=\mu^{*}$, or $\mu \in\left(\mu^{*}, \infty\right)$, respectively. This is our second result for this paper. For proofs, we employ global continuation theorem and fixed point index theory based on a weighted space as the solution space.

It is interesting to see whether the exponent $l=p$ is critical or not in the sense of existence of positive radial solutions. We end by answering this question that if $l \leq p$, then problem $(P)$ $+\left(D_{1}\right)$ does not have a positive radial solution.

Questions for global results or critical sense of exponent for existence of problem $(P)+$ $\left(D_{2}\right)$ are not answered in this work, so we leave them to the readers. A partial answer to the question for the nonexistence results to problem $(P)+\left(D_{2}\right)$ is known in $[2,3]$.

This paper is organized as follows. In Section 2, we introduce well-known theorems such as the global continuation theorem, the generalized Picone identity, and a fixed point index theorem for the index computation. In Section 3, we introduce several transformations to obtain equivalent one-dimensional $p$-Laplacian problems and also prove the existence of unbounded continuum of positive solutions using the global continuation theorem. In Section 4, figuring the shape of the unbounded continuum in Section 3, we get the existence, nonexistence, and multiplicity of solutions introduced as the partial result. In Section 5, introducing weighted spaces, we improve the result in Section 4 to a global one. In Section 6, we prove a nonexistence result which gives, in some sense, a critical exponent of existence and nonexistence.

\section{Preliminaries}

In this section, we give some known theorems which will be used in the following sections.

Theorem 2.1 (see [4, the global continuation theorem]). Let X be a Banach space and $K$ an order cone in X. Consider

$$
x=H(\mu, x),
$$


where $\mu \in \mathbb{R}_{+}$and $x \in K$. If $H: \mathbb{R}_{+} \times K \rightarrow K$ is completely continuous and $H(0, x)=0$ for all $x \in K$. Then $\mathcal{C}_{+}(K)$, the component of the solution set of $(2.1)$ containing $(0,0)$, is unbounded.

Theorem 2.2 (see [5, the generalized Picone identity]). Define

$$
\begin{aligned}
l_{p}[y] & =\left(\varphi_{p}\left(y^{\prime}\right)\right)^{\prime}+b_{1}(t) \varphi_{p}(y), \\
L_{p}[z] & =\left(\varphi_{p}\left(z^{\prime}\right)\right)^{\prime}+b_{2}(t) \varphi_{p}(z) .
\end{aligned}
$$

If $y$ and $z$ are any functions such that $y, z, b_{1} \varphi_{p}\left(y^{\prime}\right), b_{2} \varphi_{p}\left(z^{\prime}\right)$ are differentiable on $I$ and $z(t) \neq 0$ for $t \in I$, the generalized Picone identity can be written as

$$
\begin{aligned}
& \frac{d}{d t}\left\{\frac{|y|^{p} \varphi_{p}\left(z^{\prime}\right)}{\varphi_{p}(z)}-y \varphi_{p}\left(y^{\prime}\right)\right\} \\
& \quad=\left(b_{1}-b_{2}\right)|y|^{p}-\left[\left|y^{\prime}\right|^{p}+(p-1)\left|\frac{y z^{\prime}}{z}\right|^{p}-p \varphi_{p}(y) y^{\prime} \varphi_{p}\left(\frac{z^{\prime}}{z}\right)\right]-y l_{p}(y)+\frac{|y|^{p}}{\varphi_{p}(z)} L_{p}(z) .
\end{aligned}
$$

Remark 2.3. By Young's inequality, we get

$$
\left|y^{\prime}\right|^{p}+(p-1)\left|\frac{y z^{\prime}}{z}\right|^{p}-p \varphi_{p}(y) \varphi_{p}\left(\frac{z^{\prime}}{z}\right) \geq 0
$$

and the equality holds if and only if $\operatorname{sgn} y^{\prime}=\operatorname{sgn} z^{\prime}$ and $\left|y^{\prime} / y\right|^{p}=\left|z^{\prime} / z\right|^{p}$.

Theorem 2.4 (see [6]). Let $X$ be a Banach space, $K$ a cone in $X$, and $\mathcal{O}$ bounded open in $X$. Let $0 \in \mathcal{O}$ and $A: K \cap \overline{\mathcal{O}} \rightarrow K$ be condensing. Suppose that $A x \neq v x$ for all $x \in K \cap \partial \mathcal{O}$ and all $v \geq 1$. Then, $i(A, K \cap \mathcal{O}, K)=1$.

\section{The existence of unbounded continuum}

In this section, we introduce several transformations to obtain one-dimensional $p$-Laplacian problems which we will mainly analyze and then we prove the existence of unbounded continuum of positive solutions of the problem using the global continuation theorem. By consecutive changes of variables, $r=|x|, u(r)=u(|x|)$, and $t=\left(r / r_{0}\right)^{-(N-p) /(p-1)}, z(t)=u(r)$, problem $(P)+\left(D_{1}\right)$ is equivalently written as

$$
\begin{gathered}
\varphi_{p}\left(z^{\prime}\right)^{\prime}+h(t) z^{q}=0, \quad t \in(0,1), \\
z(0)=\mu>0, \quad z(1)=0,
\end{gathered}
$$

where $\varphi_{p}(s)=|s|^{p-2} s, p>1$, and $h$ is given by

$$
h(t)=\left(\frac{p-1}{N-p}\right)^{p} r_{0}^{p} t^{-p(N-1) /(N-p)} K\left(r_{0} t^{-(p-1) /(N-p)}\right) .
$$


We notice that $h$ is singular at $t=0$ and by condition $(K), h$ satisfies

$$
\left(H_{1}\right) \int_{0}^{1} s^{r} h(s) d s<\infty \text {, for some } \gamma<p-1 \text {. }
$$

For more general consideration, we assume that the coefficient function $h$ may be singular at $t=0$ and/or 1 which satisfies

$$
\text { (H) } \int_{0}^{1 / 2} \varphi_{p}^{-1}\left(\int_{s}^{1 / 2} h(\tau) d \tau\right) d s+\int_{1 / 2}^{1} \varphi_{p}^{-1}\left(\int_{1 / 2}^{s} h(\tau) d \tau\right) d s<\infty .
$$

Obviously, we see that condition $\left(H_{1}\right)$ implies condition $(H)$. Introducing $u(t)=z(t) / \mu$, we can rewrite problem (3.1) as

$$
\begin{gathered}
\varphi_{p}\left(u^{\prime}\right)^{\prime}+\lambda h(t) u^{q}=0, \quad t \in(0,1), \\
u(0)=1, \quad u(1)=0,
\end{gathered}
$$

where $\lambda=\mu^{q-p+1}$. Problems (3.1) and (3.3) share the same bifurcation phenomena with respect to $\mu$ and $\lambda$, respectively.

Similarly, if we use transformation $t=1-\left(r / r_{0}\right)^{-(N-p) /(p-1)}$, then problem $(P)+\left(D_{2}\right)$ is also written as (3.1) with $h$ given by

$$
h(t)=\left(\frac{p-1}{N-p}\right)^{p} r_{0}^{p}(1-t)^{-p(N-1) /(N-p)} K\left(r_{0}(1-t)^{-(p-1) /(N-p)}\right) .
$$

Notice that $h$ is singular at $t=1$ and by the condition $(K), h$ satisfies

$$
\left(H_{2}\right) \int_{0}^{1}(1-s)^{\gamma} h(s) d s<\infty \text {, for some } \gamma<p-1 .
$$

We see that condition $\left(H_{2}\right)$ implies condition $(H)$, and thus, for radial problem $(P)+\left(D_{2}\right)$, it is also enough to consider problem (3.3) with $h$ satisfying $(H)$. Since both problems $(P)$ $+\left(D_{i}\right), i=1,2$, can be transformed to the form (3.3), we will mainly consider problem $\left(P_{\mathcal{\lambda}}\right)$ given as follows for more general arguments:

$$
\begin{gathered}
\varphi_{p}\left(u^{\prime}(t)\right)^{\prime}+\lambda h(t) f(u(t))=0, \quad t \in(0,1), \\
u(0)=a>0, \quad u(1)=0,
\end{gathered}
$$

where $\lambda$ is a positive real parameter and $f \in C\left(\mathbb{R}_{+}, \mathbb{R}_{+}\right)$with $\mathbb{R}_{+}=[0, \infty) . h \in C\left((0,1), \mathbb{R}^{+}\right)$may be singular at $t=0$ and/or $t=1$. Let us assume the following condition:

$\left(F_{1}\right) f(u)>0$, for all $u>0$.

To fulfill conditions in the global continuation theorem, we need to consider problems with Dirichlet boundary condition. For this, we substitute $v(t)=u(t)-a(1-t)$ to get the following equivalent problem:

$$
\begin{gathered}
\varphi_{p}\left(v^{\prime}(t)-a\right)^{\prime}+\lambda h(t) f(v(t)+a(1-t))=0, \quad t \in(0,1) \\
v(0)=0=v(1) .
\end{gathered}
$$


Denote $K=\left\{u \in C_{0}[0,1]: u\right.$ is concave $\}$. Then, it is easy to see that $K$ is an order cone. Let us define operator $H: \mathbb{R}_{+} \times K \rightarrow C[0,1]$ as follows:

$$
H(\lambda, v)(t)= \begin{cases}\int_{0}^{t} \varphi_{p}^{-1}\left(\int_{s}^{A_{\lambda, v}} \operatorname{lh}(\tau) f(v(\tau)+a(1-\tau)) d \tau-\varphi_{p}(a)\right) d s+a t, & \text { if } 0 \leq t \leq A_{\lambda, v}, \\ \int_{t}^{1} \varphi_{p}^{-1}\left(\int_{A_{\lambda, v}}^{s} \lambda h(\tau) f(v(\tau)+a(1-\tau)) d \tau+\varphi_{p}(a)\right) d s-a(1-t), & \text { if } A_{\lambda, v} \leq t \leq 1,\end{cases}
$$

where

$$
\begin{aligned}
& \int_{0}^{A_{\lambda, v}} \varphi_{p}^{-1}\left(\int_{s}^{A_{\lambda, v}} \lambda h(\tau) f(v(\tau)+a(1-\tau)) d \tau-\varphi_{p}(a)\right) d s+a A_{\lambda, v} \\
& \quad=\int_{A_{\lambda, v}}^{1} \varphi_{p}^{-1}\left(\int_{A_{\lambda, v}}^{s} \lambda h(\tau) f(v(\tau)+a(1-\tau)) d \tau+\varphi_{p}(a)\right) d s-a\left(1-A_{\lambda, v}\right) .
\end{aligned}
$$

Then by condition $(H)$ and the definition of $A_{\lambda, v}$, we can easily see that $H$ is well defined and $H\left(\mathbb{R}_{+} \times K\right) \subset K$. Furthermore, $u$ is a positive solution of $\left(\widehat{P}_{\lambda}\right)$ if and only if $u=H(\lambda, u)$ on $K$. We can easily see that $H$ is completely continuous on $\mathbb{R}_{+} \times K$. The proof basically follows on the lines of Lemmas 2 and 3 in [7]. Since $H(0, u)=0$ for all $u \in K$ and $H(\lambda, 0) \neq 0$ for $\lambda>0$, as an application of Theorem 2.1, we have unbounded continuum of solutions as follows.

Theorem 3.1. Assume that $(H)$ and $\left(F_{1}\right)$ hold. Then, there exists an unbounded continuum $\widehat{C}$ bifurcating from $(0,0)$ in the closure of the set of positive solutions of $\left(\widehat{P}_{\lambda}\right)$ in $\mathbb{R}_{+} \times K$.

Corollary 3.2. Assume that $(H)$ and $\left(F_{1}\right)$ hold. Then, there exists an unbounded continuum $C$ bifurcating from $\left(0, u_{0}\right)$, where $u_{0}(t)=a(1-t)$, in the closure of the set of positive solutions of $\left(P_{\lambda}\right)$ in $\mathbb{R}_{+} \times K$.

\section{The shape of continuum}

In this section, we will figure the shape of unbounded subcontinuum $\mathcal{C}$ of positive solutions of problem $\left(P_{\lambda}\right)$ known to exist by Corollary 3.2:

$$
\begin{gathered}
\varphi_{p}\left(u^{\prime}(t)\right)^{\prime}+\lambda h(t) f(u(t))=0, \quad t \in(0,1), \\
u(0)=a>0, \quad u(1)=0,
\end{gathered}
$$

where $f \in C\left(\mathbb{R}_{+}, \mathbb{R}_{+}\right), h \in C\left((0,1), \mathbb{R}^{+}\right)$. We assume an additional condition for this section:

$\left(F_{2}\right) f_{\infty} \triangleq \lim _{u \rightarrow \infty}\left(f(u) / u^{p-1}\right)=\infty$.

Using the generalized Picone identity and the properties of the $p$-sine function $[8,9]$, we obtain the following lemma.

Lemma 4.1. Assume that $\left(F_{1}\right)$ and $\left(F_{2}\right)$ hold. Let $u$ be a positive solution of $\left(P_{\lambda}\right)$. Then, there exists $\bar{\lambda}>0$ such that $\lambda \leq \bar{\lambda}$. 
Proof. Since $u$ is concave and $u(0)=a, u(t) \geq(1 / 4) a$, for all $t \in(0,3 / 4)$, then it follows from $\left(F_{1}\right)$ and $\left(F_{2}\right)$ that there exists $b>0$ such that $f(u)>b u^{p-1}$, for $u \geq(1 / 4) a$. This implies

$$
0=\varphi_{p}\left(u^{\prime}(t)\right)^{\prime}+\lambda h(t) f(u(t))>\varphi_{p}\left(u^{\prime}(t)\right)+\lambda b h(t) \varphi_{p}(u(t)), \quad t \in\left(0, \frac{3}{4}\right) .
$$

Putting $m:=\min _{t \in[1 / 4,3 / 4]} h(t)>0$, we have

$$
\varphi_{p}\left(u^{\prime}(t)\right)^{\prime}+\lambda b m \varphi_{p}(u(t))<0, \quad t \in\left(\frac{1}{4}, \frac{3}{4}\right) .
$$

It is easy to check that $w(t)=S_{q}\left(2 \pi_{p}(t-1 / 4)\right)$ is a solution of

$$
\begin{gathered}
\varphi_{p}\left(w^{\prime}(t)\right)^{\prime}+\left(2 \pi_{p}\right)^{p} \varphi_{p}(w(t))=0, \quad t \in\left(\frac{1}{4}, \frac{3}{4}\right), \\
w\left(\frac{1}{4}\right)=0=w\left(\frac{3}{4}\right),
\end{gathered}
$$

where $S_{q}$ is the $q$-sine function with $1 / p+1 / q=1$ and $\pi_{p}=2 \pi(p-1)^{1 / p} / p \sin (\pi / p)$. Taking $y=w, z=u, b_{1}=\left(2 \pi_{p}\right)^{p}$, and $b_{2}=\lambda b m$ in (2.3) and integrating from $1 / 4$ to $3 / 4$, we have

$$
\int_{1 / 4}^{3 / 4}\left(\left(2 \pi_{p}\right)^{p}-\lambda b m\right)|w|^{p} d t \geq 0 .
$$

Thus,

$$
\lambda \leq \frac{\left(2 \pi_{p}\right)^{p}}{b m}:=\bar{\lambda}
$$

Lemma 4.2. Assume that $\left(F_{2}\right)$ holds. Let $J$ be a compact interval in $(0, \infty)$. Then, for all $\lambda \in J$, there exists $M_{J}>0$ such that all possible positive solutions $u$ of $\left(P_{\lambda}\right)$ satisfy $\|u\|_{\infty} \leq M_{J}$.

Proof. Suppose on the contrary that there exists a sequence $\left(u_{n}\right)$ of positive solutions of $\left(P_{\lambda_{n}}\right)$ with $\left(\lambda_{n}\right) \subset J \triangleq[\alpha, \beta]$ and $\left\|u_{n}\right\|_{\infty} \rightarrow \infty$ as $n \rightarrow \infty$. It follows from the concavity of $u_{n}$ that

$$
u_{n}(t) \geq \frac{1}{4}\left\|u_{n}\right\|_{\infty}
$$

for all $t \in(1 / 4,3 / 4)$. Take $M=2\left(\left(2 \pi_{p}\right)^{p} / \alpha m\right)$ with $m=\min _{t \in[1 / 4,3 / 4]} h(t)>0$. By $\left(F_{2}\right)$, there exists $K>0$ such that $f(u)>M \varphi_{p}(u)$, for all $u>K$. From the assumption, we get $\left\|u_{N}\right\|_{\infty}>4 K$ for sufficiently large $N$. Therefore by (4.6), we have

$$
f\left(u_{N}(t)\right)>M \varphi_{p}\left(u_{N}(t)\right), \quad t \in\left(\frac{1}{4}, \frac{3}{4}\right) .
$$

Hence, we have

$$
\varphi_{p}\left(u_{N}^{\prime}(t)\right)^{\prime}+\alpha M m \varphi_{p}\left(u_{N}(t)\right)<0, \quad t \in\left(\frac{1}{4}, \frac{3}{4}\right) .
$$

As in the proof of Lemma 4.1, for $w(t)=S_{q}\left(2 \pi_{p}(t-1 / 4)\right)$, taking $y=w, z=u, b_{1}=\left(2 \pi_{p}\right)^{p}$, and $b_{2}=\alpha M m$ in (2.3), we obtain

$$
M \leq \frac{\left(2 \pi_{p}\right)^{p}}{\alpha m} .
$$

This is a contradiction. 
We now state and prove the main theorem in this section.

Theorem 4.3. Assume that $(H),\left(F_{1}\right)$, and $\left(F_{2}\right)$ hold. Then, there exist $0<\lambda^{*} \leq \lambda_{*}$ such that $\left(P_{\lambda}\right)$ has two positive solutions for $0<\lambda<\lambda^{*}$, one positive solution for $\lambda^{*} \leq \lambda \leq \lambda_{*}$, and no positive solution for $\lambda>\lambda_{*}$.

Proof. Define $\lambda^{*}:=\sup \left\{\mu\right.$ : problem $\left(P_{\lambda}\right)$ has at least two positive solutions for all $\left.\lambda \in(0, \mu)\right\}$. Then by Lemmas 4.1 and $4.2, \lambda^{*}<\infty$. Suppose that there exists $\hat{\lambda} \geq \lambda^{*}$ such that $\left(P_{\widehat{\lambda}}\right)$ has a positive solution, say $\widehat{u}$, that is,

$$
\begin{gathered}
\varphi_{p}\left(\widehat{u}^{\prime}(t)-a\right)^{\prime}+\widehat{\jmath} h(t) f(\widehat{u}(t)+a(1-t))=0, \quad t \in(0,1), \\
\widehat{u}(0)=0=\widehat{u}(1) .
\end{gathered}
$$

For fixed $\lambda \in(0, \widehat{\lambda})$, define $\bar{T}_{\lambda}: C[0,1] \rightarrow C[0,1]$ by

$$
\bar{T}_{\lambda}(u)(t)= \begin{cases}\int_{0}^{t} \varphi_{p}^{-1}\left(\int_{s}^{B} \lambda h(\tau) f(\gamma(u(\tau))-a(1-\tau)) d \tau-\varphi_{p}(a)\right) d s+a t, & 0 \leq t \leq B, \\ \int_{t}^{1} \varphi_{p}^{-1}\left(\int_{B}^{s} \lambda h(\tau) f(\gamma(u(\tau))-a(1-\tau)) d \tau+\varphi_{p}(a)\right) d s-a(1-t), & B \leq t \leq 1,\end{cases}
$$

where $\gamma: \mathbb{R} \rightarrow \mathbb{R}$ is defined by

$$
r(u)= \begin{cases}\widehat{u}(t), & \text { if } u>\widehat{u}(t) \\ u, & \text { if } 0 \leq u \leq \widehat{u}(t) \\ 0, & \text { if } u<0\end{cases}
$$

and $B$ satisfies

$$
\begin{aligned}
& \int_{0}^{B} \varphi_{p}^{-1}\left(\int_{s}^{B} \lambda h(\tau) f(\gamma(u(\tau))-a(1-\tau)) d \tau-\varphi_{p}(a)\right) d s+a B \\
& \quad=\int_{B}^{1} \varphi_{p}^{-1}\left(\int_{B}^{s} \lambda h(\tau) f(\gamma(u(\tau))-a(1-\tau)) d \tau+\varphi_{p}(a)\right) d s-a(1-B) .
\end{aligned}
$$

It is easy to check that $\bar{T}_{\mathcal{\lambda}}$ is completely continuous on $C[0.1]$. Let us consider the following modified problem:

$$
\begin{gathered}
\varphi_{p}\left(u^{\prime}(t)-a\right)^{\prime}+\lambda h(t) f(\gamma(u(t))+a(1-t))=0, \quad t \in(0,1), \\
u(0)=0=u(1) .
\end{gathered}
$$

Then, solution $u$ of $\left(M_{\curlywedge}\right)$ is concave and nontrivial. It follows from the definition of $\gamma$ and the continuity of $f$ that there exists $R_{1}>0$ such that $\left\|\bar{T}_{\lambda} u\right\|_{\infty}<R_{1}$ for all $u \in C[0,1]$. Then, by 
Schauder's fixed point theorem there exists $u \in C[0,1]$ such that $\bar{T}_{\curlywedge} u=u$. Hence, $u$ is a positive solution of $\left(M_{\curlywedge}\right)$.

We claim that $u(t) \leq \widehat{u}(t)$, for all $t \in[0,1]$. If the claim is not true, then there exists an interval $\left[t_{1}, t_{2}\right] \subset[0,1]$ such that $u\left(t_{1}\right)=\widehat{u}\left(t_{1}\right), u\left(t_{2}\right)=\widehat{u}\left(t_{2}\right)$, and $u(t)>\widehat{u}(t)$, for all $t \in\left(t_{1}, t_{2}\right)$. Put $\alpha(t)=u(t)-\widehat{u}(t)$. Then, $\alpha\left(t_{1}\right)=0=\alpha\left(t_{2}\right)$ and there exists an interval $[b, c] \subset\left[t_{1}+\delta, t_{2}-\delta\right]$ such that $\alpha^{\prime}(b)=u^{\prime}(b)-\widehat{u}^{\prime}(b)>0$ and $\alpha^{\prime}(c)=u^{\prime}(c)-\widehat{u}^{\prime}(c)<0$ for sufficiently small $\delta>0$. Therefore, we have

$$
u^{\prime}(b)-a>\widehat{u}^{\prime}(b)-a, \quad u^{\prime}(c)-a<\widehat{u}^{\prime}(c)-a .
$$

It follows from the monotonicity of $\varphi_{p}$ that

$$
\varphi_{p}\left(u^{\prime}(b)-a\right)>\varphi_{p}\left(\hat{u}^{\prime}(b)-a\right), \quad \varphi_{p}\left(u^{\prime}(c)-a\right)<\varphi_{p}\left(\hat{u}^{\prime}(c)-a\right) .
$$

Since $0<\lambda<\hat{\imath}$, we get

$$
\begin{aligned}
0 & >\varphi_{p}\left(u^{\prime}(c)-a\right)-\varphi_{p}\left(\widehat{u}^{\prime}(c)-a\right)-\varphi_{p}\left(u^{\prime}(b)-a\right)+\varphi_{p}\left(\widehat{u}^{\prime}(b)-a\right) \\
& =\left[\varphi_{p}\left(u^{\prime}(c)-a\right)-\varphi_{p}\left(u^{\prime}(b)-a\right)\right]-\left[\varphi_{p}\left(\widehat{u}^{\prime}(c)-a\right)-\varphi_{p}\left(\widehat{u}^{\prime}(b)-a\right)\right] \\
& =\int_{b}^{c}\left\{\left[\varphi_{p}\left(u^{\prime}(t)-a\right)\right]^{\prime}-\left[\varphi_{p}\left(\widehat{u}^{\prime}(t)-a\right)\right]^{\prime}\right\} d t \\
& =\int_{b}^{c}\{-\lambda h(t) f(\gamma(u(t))+a(1-t))+\widehat{\lambda} h(t) f(\widehat{u}(t)+a(1-t))\} d t \\
& =(\widehat{\lambda}-\lambda) \int_{b}^{c} h(t) f(\widehat{u}(t)+a(1-t)) d t>0 .
\end{aligned}
$$

This contradiction implies $u(t) \leq \widehat{u}(t)$, for all $t \in[0,1]$. Therefore, by the definition of $\gamma, u$ turns out a positive solution of $\left(P_{\lambda}\right)$. Define $\lambda_{*}=\sup \left\{\lambda:\left(P_{\lambda}\right)\right.$ has at least one positive solution $\}$. Then by Lemma $4.1, \lambda_{*}<\infty$. Furthermore by Lemma 4.2 and compactness of $H$, we can show that $\left(P_{\lambda_{*}}\right)$ has a positive solution in frame of standard limit argument and this completes the proof.

Take $f(u)=u^{q}, q>p-1$, in problem $\left(P_{\lambda}\right)$, then by the transformation arguments in Section 3, solutions of $\left(P_{\lambda}\right)$ correspond to those of problem (3.1) which is the radial problem of $(P)+\left(D_{i}\right), i=1,2$. In this case, conditions $\left(F_{i}\right), i=1,2$, in Theorem 4.3 are redundant and we get the following corollary.

Corollary 4.4. Assume $q>p-1$ and assume that

(K) there exists $\beta>p-1$ such that $\int_{r_{0}}^{\infty} r^{\beta} K(r) d r<\infty$.

Then, there exist $\mu_{0} \geq \mu^{*}>0$ such that $(P)+\left(D_{i}\right), i=1,2$, has at least two positive radial solutions for $\mu \in\left(0, \mu^{*}\right)$, at least one positive radial solution for $\mu \in\left[\mu^{*}, \mu_{0}\right]$, and no positive radial solution for $\mu \in\left(\mu_{0}, \infty\right)$. 


\section{Global existence result}

Multiplicity of solutions on $\left[\lambda^{*}, \lambda_{*}\right]$ is not known in Theorem 4.3. Analytic difficulty on this range is caused by lack of topological properties in solution space $C[0,1]$, mainly lack of controllability of derivatives of solutions at the boundary. In this section, we overcome this difficulty by employing a weighted space as new solution space specially for problem $(P)+$ $\left(D_{1}\right)$. For this purpose, let us consider the following $p$-Laplacian problem:

$$
\begin{gathered}
\operatorname{div}\left(|\nabla u|^{p-2} \nabla u\right)+|x|^{-l} u^{q}=0 \quad \text { in }|x|>r_{0} \\
\left.u\right|_{\partial \Omega}=0, \quad u \longrightarrow \mu>0 \quad \text { as }|x| \longrightarrow \infty
\end{gathered}
$$

We notice that $K(|x|)=|x|^{-l}$ with $l>p$ satisfies condition $(K)$. By transformations $r=$ $|x|, u(r)=u(|x|)$, and $t=\left(r / r_{0}\right)^{-(N-p) /(p-1)}, u(r)=z(t)$, we obtain

$$
\begin{gathered}
\varphi_{p}\left(z^{\prime}(t)\right)^{\prime}+\left(\frac{p-1}{N-p}\right)^{p} r_{0}^{p} t^{(-p(N-1)+(p-1) l) /(N-p)} z^{q}(t)=0, \quad t \in(0,1), \\
z(0)=\mu>0, \quad z(1)=0 .
\end{gathered}
$$

For $\alpha=(p(N-1)-(p-1) l) /(N-p)$, condition $l>p$ corresponds to $\alpha<p$. By another transformation $u(t)=z(t) / \mu$, the above problem can be transformed into

$$
\begin{gathered}
\varphi_{p}\left(u^{\prime}(t)\right)^{\prime}+\lambda t^{-\alpha} u^{q}(t)=0, \quad t \in(0,1), \\
u(0)=1>0, \quad u(1)=0,
\end{gathered}
$$

where $\lambda=\mu^{q-p+1}$.

As in Section 3, we consider problem $\left(E_{\curlywedge}\right)$ given as follows for more general arguments:

$$
\begin{gathered}
\varphi_{p}\left(u^{\prime}(t)\right)^{\prime}+\lambda t^{-\alpha} f(u(t))=0, \quad t \in(0,1), \\
u(0)=a>0, \quad u(1)=0,
\end{gathered}
$$

where $\lambda$ is a positive real parameter and $f \in C\left(\mathbb{R}_{+}, \mathbb{R}_{+}\right)$. We give an additional assumption in this section:

$\left(F_{3}\right) f$ is nondecreasing.

Now the aim of our work here is to investigate bifurcation phenomena of positive solutions for problem $\left(E_{\curlywedge}\right)$. Again introducing $v(t)=u(t)-a(1-t)$, we rewrite $\left(E_{\mathcal{\lambda}}\right)$ to the following equivalent Dirichlet boundary problem:

$$
\begin{gathered}
\varphi_{p}\left(v^{\prime}(t)-a\right)^{\prime}+\lambda t^{-\alpha} f(v(t)+a(1-t))=0, \quad t \in(0,1), \\
v(0)=0=v(1) .
\end{gathered}
$$

We first state the main theorem in this section.

Theorem 5.1. Assume that $\left(F_{1}\right),\left(F_{2}\right)$, and $\left(F_{3}\right)$ hold. Also assume that $\alpha<p$. Then, there exists $\lambda^{*}>0$ such that $\left(\widehat{E}_{\lambda}\right)$ has at least two positive solutions for $\lambda \in\left(0, \lambda^{*}\right)$, at least one positive solution for $\lambda=\lambda^{*}$, and no positive solution for $\lambda \in\left(\lambda^{*}, \infty\right)$. 
If $\alpha<1$, then $h(t)=t^{-\alpha}$ is of class $L^{1}(0,1]$. Thus, solution space is $C^{1}[0,1]$ and by typical Leray-Schauder degree argument in the frame of $C^{1}$-topology, we can prove that the theorem is true (see, [10]). Therefore, in this section, we focus on the case $1 \leq \alpha<p$. Define

$$
w(t)= \begin{cases}t^{(\alpha-1) /(p-1)}, & \text { if } 1<\alpha<p, \\ \min \left\{(-\ln t)^{-1 /(p-1)}, 1\right\}, & \text { if } \alpha=1 .\end{cases}
$$

We can easily know that

$$
\lim _{t \rightarrow 0^{+}} w(t)=0, \quad 0<w(t) \leq 1, t \in(0,1], w^{-1} \in L^{1}(0,1) .
$$

The following lemma is essential to introduce our weighted space $C_{w}[0,1]$ and very useful to construct a bounded open set of solutions in the space for fixed point index computation.

Lemma 5.2. If $u$ is a solution of $\left(\widehat{E}_{\mathcal{\lambda}}\right)$, then $w u^{\prime} \in C(0,1]$ and

$$
0<\lim _{t \rightarrow 0^{+}}\left(w u^{\prime}\right)(t)<\infty .
$$

Proof. Let $u$ be a solution of $\left(\widehat{E}_{\curlywedge}\right)$. Then, we have

$$
u^{\prime}(t)=\varphi_{p}^{-1}\left(\lambda \int_{t}^{A} \tau^{-\alpha} f(u(\tau)+a(1-\tau)) d \tau-\varphi_{p}(a)\right)+a,
$$

where $u^{\prime}(A)=0$. Since $w \in C(0,1]$ and $u^{\prime} \in C(0,1]$, we only need to show

$$
0<\lim _{t \rightarrow 0^{+}}\left(w u^{\prime}\right)(t)<\infty .
$$

In fact, if $1<\alpha<p$, then by L'Hospital's rule and $\lim _{t \rightarrow 0^{+}} w(t)=0$, we have

$$
\begin{aligned}
\lim _{t \rightarrow 0^{+}}\left(w u^{\prime}\right)(t) & =\lim _{t \rightarrow 0^{+}}\left(\varphi_{p}^{-1}\left[\frac{\lambda \int_{t}^{A} \tau^{-\alpha} f(u(\tau)+a(1-\tau)) d \tau-\varphi_{p}(a)}{t^{1-\alpha}}\right]+a w(t)\right) \\
& =\varphi_{p}^{-1}\left(\lambda \frac{1}{\alpha-1} f(a)\right)>0 .
\end{aligned}
$$

If $\alpha=1$, then similarly we may obtain

$$
\lim _{t \rightarrow 0^{+}}\left(w u^{\prime}\right)(t)=\varphi_{p}^{-1}(\lambda f(a))>0 .
$$

Thus, the proof is complete.

Define $C_{w}[0,1]=\left\{u \in C_{0}[0,1] \cap C^{1}(0,1]: \lim _{t \rightarrow 0^{+}}\left(w u^{\prime}\right)(t)\right.$ exists $\}$. We notice that if $u \in C_{w}[0,1]$, then there exists an extension $\overline{w u^{\prime}} \in C[0,1]$ of $w u^{\prime}$ such that

$$
\overline{w u^{\prime}}(t)= \begin{cases}\lim _{t \rightarrow 0^{+}}\left(w u^{\prime}\right)(t), & t=0 \\ \left(w u^{\prime}\right)(t), & t \in(0,1]\end{cases}
$$

Define

$$
\|u\|_{w}=\|u\|_{\infty}+\left\|\overline{w u^{\prime}}\right\|_{\infty} .
$$


Proposition 5.3. $\left(C_{w}[0,1],\|\cdot\|_{w}\right)$ is a Banach space.

Proof. It is easy to see that $C_{w}[0,1]$ is a normed linear space. We only need to check that $C_{w}[0,1]$ is complete. In fact, let $\left(u_{n}\right)$ be a Cauchy sequence in $C_{w}[0,1]$. That is, $\left(u_{n}\right)$ is a Cauchy sequence in $C_{0}[0,1]$ and $\left(\overline{w u_{n}^{\prime}}\right)$ is a Cauchy sequence in $C[0,1]$. Since both $C_{0}[0,1]$ and $C[0,1]$ are Banach spaces, there exist $u \in C_{0}[0,1]$ and $v \in C[0,1]$ such that $u_{n} \rightarrow u$ in $C_{0}[0,1]$ and $\overline{w u_{n}^{\prime}} \rightarrow v$ in $C[0,1]$. Since $w(t)>0$, for $t \in(0,1]$, there exists $v_{1} \in C(0,1]$ such that $v(t)=w(t) v_{1}(t)$, for all $t \in(0,1]$. For $\delta>0$, we know $u_{n}^{\prime} \rightarrow v_{1}$ in $C[\delta, 1]$. This implies $v_{1} \equiv u^{\prime}$ in $[\delta, 1]$. Since $\delta>0$ is arbitrary, $w u_{n}^{\prime} \rightarrow w u^{\prime}$ pointwise in $t \in(0,1]$. Therefore, by the uniqueness of limit, $w u^{\prime} \equiv v$ on $(0,1]$. Since $\overline{w u_{n}^{\prime}} \rightarrow v$ in $C[0,1]$, we have

$$
\begin{aligned}
v(0) & =\lim _{n \rightarrow \infty} \overline{w u_{n}^{\prime}}(0) \\
& =\lim _{n \rightarrow \infty} \lim _{t \rightarrow 0^{+}} w u_{n}^{\prime}(t) \\
& =\lim _{t \rightarrow 0^{+}} \lim _{n \rightarrow \infty} w u_{n}^{\prime}(t) \\
& =\lim _{t \rightarrow 0^{+}} w u^{\prime}(t) .
\end{aligned}
$$

Therefore, $u \in C_{w}[0,1]$ and $\overline{w u^{\prime}} \equiv v$ on $[0,1]$. This implies $u_{n} \rightarrow u$ in $C_{w}[0,1]$ and the proof is complete.

Let $K=\left\{u \in C_{w}[0,1] \mid u\right.$ is concave on $\left.(0,1)\right\}$. Then, it is easy to check that $K$ is an order cone. Define $H: \mathbb{R}_{+} \times K \rightarrow C_{w}[0,1]$ by

$$
H(\lambda, u)(t) \triangleq \begin{cases}\int_{0}^{t} \varphi_{p}^{-1}\left(\int_{s}^{A_{\lambda, u}} \lambda \tau^{-\alpha} f(u(\tau)+a(1-\tau)) d \tau-\varphi_{p}(a)\right) d s+a t, & 0 \leq t \leq A_{\lambda, u}, \\ \int_{t}^{1} \varphi_{p}^{-1}\left(\int_{A_{\lambda, u}}^{s} \lambda \tau^{-\alpha} f(u(\tau)+a(1-\tau)) d \tau+\varphi_{p}(a)\right) d s-a(1-t), & A_{\lambda, u} \leq t \leq 1,\end{cases}
$$

where

$$
\begin{aligned}
\int_{0}^{A_{\lambda, u}} & \varphi_{p}^{-1}\left(\int_{s}^{A_{\lambda, u}} \lambda \tau^{-\alpha} f(u(\tau)+a(1-\tau)) d \tau-\varphi_{p}(a)\right) d s+a A_{\curlywedge, u} \\
\quad= & \int_{A_{\lambda, u}}^{1} \varphi_{p}^{-1}\left(\int_{A_{\lambda, u}}^{s} \lambda \tau^{-\alpha} f(u(\tau)+a(1-\tau)) d \tau+\varphi_{p}(a)\right) d s-a\left(1-A_{\lambda, u}\right) .
\end{aligned}
$$

Assume that $\left(F_{1}\right)$ holds. Then, by the similar argument in the proof of Lemma 5.2 and the definition of $H$, we see that $H$ is well defined and $H\left(\mathbb{R}_{+} \times K\right) \subset K$. Furthermore, $u$ is a positive solution of $\left(\widehat{E}_{\lambda}\right)$ if and only if $u=H(\lambda, u)$ on $K$. The following lemma can be proved by standard argument and we skip the proof. 
Lemma 5.4. Assume that $\left(F_{1}\right)$ holds. Then, $H$ is completely continuous on $\mathbb{R}_{+} \times K$.

Since $H(0, u)=0$, for all $u \in K$ and $H(\lambda, 0) \neq 0$, for $\lambda>0$, Lemma 5.4 and the global continuation theorem imply that there exists an unbounded continuum $\mathcal{C}$ of positive solutions of $\left(\widehat{E}_{\curlywedge}\right)$ bifurcating from $(0,0)$.

Lemma 5.5. Assume that $\left(F_{1}\right)$ and $\left(F_{2}\right)$ hold. Let $u$ be a positive solution of $\left(\widehat{E}_{\lambda}\right)$. Then, there exists $\bar{\lambda}>0$ such that $\lambda \leq \bar{\lambda}$.

Proof. Taking $h(t)=t^{-\alpha}$ with $\alpha<p$, we can prove this lemma by the same argument in the proof of Lemma 4.1.

Lemma 5.6. Assume that $\left(F_{2}\right)$ holds and let I be a compact interval in $(0, \infty)$. Then, there exists $b_{I}>0$ such that for all possible positive solution $u$ of $\left(\widehat{E}_{\lambda}\right)$ with $\lambda \in I$, one has

$$
\|u\|_{w} \leq b_{I}
$$

Proof. Assume on the contrary that there exists a sequence $\left(u_{n}\right)$ of a positive solution of $\left(\widehat{E}_{\lambda_{n}}\right)$ with $\lambda_{n} \in I$ such that $\left\|u_{n}\right\|_{w} \rightarrow \infty$ as $n \rightarrow \infty$. We claim $\left\|u_{n}\right\|_{\infty} \rightarrow \infty$ as $n \rightarrow \infty$. This contradicts Lemma 4.2 and the proof is complete. If the claim is not true, then there exists $M_{1}>0$ such that $\left\|u_{n}\right\|_{\infty} \leq M_{1}$, for all $n$. Since $u_{n}$ is a positive solution of $\left(\widehat{E}_{\lambda_{n}}\right)$, we get

$$
-\varphi_{p}\left(u_{n}^{\prime}(t)-a\right)^{\prime}=\lambda_{n} t^{-\alpha} f\left(u_{n}(t)+a(1-t)\right) \leq d t^{-\alpha}, \quad t \in(0,1),
$$

where $d=\sup I \max _{u \in\left[0, M_{1}+a\right]} f(u)$. Integrating this from $t$ to 1 , we have

$$
\begin{gathered}
\left|\varphi_{p}\left(u_{n}^{\prime}(1)-a\right)-\varphi_{p}\left(u_{n}^{\prime}(t)-a\right)\right| \leq d \int_{t}^{1} s^{-\alpha} d s, \\
\left|\varphi_{p}\left(u_{n}^{\prime}(t)-a\right)\right| \leq\left|\varphi_{p}\left(u_{n}^{\prime}(1)-a\right)\right|+d \int_{t}^{1} s^{-\alpha} d s .
\end{gathered}
$$

Since $\varphi_{p}^{-1}$ is increasing, we obtain

$$
\begin{aligned}
\left|u_{n}^{\prime}(t)-a\right| & \leq \varphi_{p}^{-1}\left[\left|\varphi_{p}\left(u_{n}^{\prime}(1)-a\right)\right|+d \int_{t}^{1} s^{-\alpha} d s\right] \\
& \leq 2^{(2-p) /(p-1)}\left[\left|u_{n}^{\prime}(1)\right|+a+\varphi_{p}^{-1}\left(d \int_{t}^{1} s^{-\alpha} d s\right)\right] .
\end{aligned}
$$

Therefore, by the similar computation in the proof of Lemma 5.4, we obtain

$$
\begin{aligned}
w(t)\left|u_{n}^{\prime}(t)\right| & \leq 2^{(2-p) /(p-1)}\left[\left|u_{n}^{\prime}(1)\right|+\left(1+2^{(p-2) /(p-1)}\right) a+\varphi_{p}^{-1}\left(d w^{p-1}(t) \int_{t}^{1} s^{-\alpha} d s\right)\right] \\
& \leq 2^{(2-p) /(p-1)}\left[\left|Y^{\prime}(1)\right|+\left(1+2^{(p-2) /(p-1)}\right) a+\varphi_{p}^{-1}\left(d \max _{t \in[0,1]}\left\{w(t)^{p-1} \int_{t}^{1} s^{-\alpha} d s\right\}\right)\right] \\
& <+\infty,
\end{aligned}
$$


where

$$
Y(t)= \begin{cases}\int_{0}^{t} \varphi_{p}^{-1}\left(d \int_{s}^{B} \tau^{-\alpha} d \tau-\varphi_{p}(a)\right) d s+a t, & 0 \leq t \leq B, \\ \int_{t}^{1} \varphi_{p}^{-1}\left(d \int_{B}^{s} \tau^{-\alpha} d \tau+\varphi_{p}(a)\right) d s-a(1-t), & B \leq t \leq 1,\end{cases}
$$

with $Y(B)=\|Y\|_{\infty}$. This implies that $\left\{\left\|w u_{n}^{\prime}\right\|_{\infty}\right\}$ is bounded. This contradicts our assumption $\left\|u_{n}\right\|_{w} \rightarrow \infty$ and the claim is proved.

Let us assume that problem $\left(\widehat{E}_{\mathcal{\lambda}}\right)$ has a positive solution at $\lambda_{*}>0$ so let $u_{*}$ be a positive solution of $\left(\widehat{E}_{\lambda_{*}}\right)$. We see that $u_{*}$ satisfies

$$
\varphi_{p}\left(u_{*}^{\prime}(t)-a\right)^{\prime}+\lambda_{*} t^{-\alpha} f\left(u_{*}(t)+a(1-t)\right)=0, \quad t \in(0,1) .
$$

Consider a fixed parameter $\lambda \in\left(0, \lambda_{*}\right)$. For $N>0$, let us define

$$
\begin{gathered}
\Omega_{N}=\left\{u \in C_{w}[0,1] \mid 0<u(t)<u_{*}(t), t \in(0,1), 0<w u^{\prime}\left(0^{+}\right)<w u_{*}^{\prime}\left(0^{+}\right),\right. \\
\left.u_{*}^{\prime}\left(1^{-}\right)<u^{\prime}\left(1^{-}\right)<0 \text { and }\left\|\overline{w u^{\prime}}\right\|_{\infty}<N\right\} .
\end{gathered}
$$

Then by Lemma 5.2, $\Omega_{N}$ is bounded and open in $C_{w}[0,1]$. Consider the following modified problem:

$$
\begin{gathered}
\varphi_{p}\left(u^{\prime}(t)-a\right)^{\prime}+\lambda t^{-\alpha} f(\gamma(u(t))+a(1-t))=0 \\
u(0)=0=u(1)
\end{gathered}
$$

where $\gamma: \mathbb{R} \rightarrow \mathbb{R}_{+}$by

$$
\gamma(u)= \begin{cases}u_{*}(t), & \text { if } u>u_{*}(t) \\ u, & \text { if } 0 \leq u \leq u_{*}(t) \\ 0, & \text { if } u<0\end{cases}
$$

Lemma 5.7. Assume that $\left(F_{1}\right),\left(F_{2}\right)$, and $\left(F_{3}\right)$ hold. If $u$ is a positive solution of $\left(M_{\lambda 1}\right)$ for $\lambda \in\left(0, \lambda_{*}\right)$, then $u \in \Omega_{N} \cap K$, for some $N>0$.

Proof. Let $u$ be a positive solution of $\left(M_{\curlywedge 1}\right)$. We first show $0<u(t) \leq u_{*}(t), t \in(0,1)$. If it is not true, there exists $\left[t_{1}, t_{2}\right] \subset[0,1]$ such that $u(t)>u_{*}(t)$ for $t \in\left(t_{1}, t_{2}\right), u\left(t_{1}\right)=u_{*}\left(t_{1}\right)$, and $u\left(t_{2}\right)=u_{*}\left(t_{2}\right)$. Since $u-u_{*} \in C_{0}\left[t_{1}, t_{2}\right]$, there exists $A \in\left(t_{1}, t_{2}\right)$ such that

$$
u^{\prime}(A)=u_{*}^{\prime}(A), \quad u(A)-u_{*}(A)>0 .
$$

Since $\gamma(u(t))+a(1-t) \leq u_{*}(t)+a(1-t), \lambda<\lambda_{*}$, and $f$ is nondecreasing, we have

$$
\lambda_{*} f\left(u_{*}(t)+a(1-t)\right)>\lambda f(\gamma(u(t))+a(1-t)) .
$$


This implies

$$
\varphi_{p}\left(u^{\prime}(t)-a\right)^{\prime}+\lambda_{*} t^{-\alpha} f\left(u_{*}(t)+a(1-t)\right)>0, \quad t \in(0,1) .
$$

From (5.21) and (5.26), we have

$$
\varphi_{p}\left(u_{*}^{\prime}(t)-a\right)^{\prime}-\varphi_{p}\left(u^{\prime}(t)-a\right)^{\prime}<0, \quad t \in(0,1) .
$$

For $t \in(A, 1)$, integrating (5.27) from $A$ to $t$, we have $u_{*}^{\prime}(t) \leq u^{\prime}(t)$. Again, integrating this from $A$ to 1 , we get

$$
u_{*}(A) \geq u(A)
$$

This contradicts (5.24).

Second, we show $u(t)<u_{*}(t), t \in(0,1)$. If it is not true, then by the first argument and (5.27), we have only one case; there exist $t_{3} \in(0,1)$ and $\delta_{1}>0$ such that $u\left(t_{3}\right)=u_{*}\left(t_{3}\right)$, $u(t)<u_{*}(t), t \in\left(t_{3}-\delta_{1}, t_{3}+\delta_{1}\right) \backslash\left\{t_{3}\right\}$, and $u^{\prime}\left(t_{3}\right)=u_{*}^{\prime}\left(t_{3}\right)$. For $t \in\left(t_{3}-\delta_{1}, t_{3}\right)$, integrating (5.27) from $t$ to $t_{3}$, we have

$$
u_{*}^{\prime}(t) \geq u^{\prime}(t), \quad \text { for } t \in\left(t_{3}-\delta_{1}, t_{3}\right)
$$

Again integrating this from $t_{3}-\delta_{1} / 2$ to $t_{3}$, we get

$$
u_{*}\left(t_{3}-\frac{\delta_{1}}{2}\right) \leq u\left(t_{3}-\frac{\delta_{1}}{2}\right)
$$

and this is a contradiction.

Third, we show that $0<\lim _{t \rightarrow 0^{+}} w u^{\prime}(t)<\lim _{t \rightarrow 0^{+}} w u_{*}^{\prime}(t)$. By the second argument, $\gamma(u(t))=u(t)$, for $t \in(0,1)$. By the similar calculation as in Lemma 5.2, we have

$$
\lim _{t \rightarrow 0^{+}} w u^{\prime}(t)=\varphi_{p}^{-1}(\lambda f(a))<\varphi_{p}^{-1}\left(\lambda_{*} f(a)\right)=\lim _{t \rightarrow 0^{+}} w u_{*}^{\prime}(t),
$$

if $\alpha=1$. The case $1<\alpha<p$ is similar.

Fourth, we show that $0>u^{\prime}(1)>u_{*}^{\prime}(1)$. We first claim that there exists $c \in(0,1)$ such that $u^{\prime}(c)>u_{*}^{\prime}(c)$. Indeed, otherwise, $u^{\prime}(t) \leq u_{*}^{\prime}(t)$, for all $t \in(0,1)$. Integrating this from $t$ to 1 , we have

$$
u(t) \geq u_{*}(t), \quad \text { for } t \in(0,1)
$$

This is a contradiction by the second argument. Integrating (5.27) from $c$ to 1 , we obtain

$$
\varphi_{p}\left(u_{*}^{\prime}(1)-a\right)-\varphi_{p}\left(u^{\prime}(1)-a\right)<\varphi_{p}\left(u_{*}^{\prime}(c)-a\right)-\varphi_{p}\left(u^{\prime}(c)-a\right)<0
$$

and thus

$$
u_{*}^{\prime}(1)<u^{\prime}(1)
$$

Since $u$ is a positive solution of $\left(M_{\curlywedge 1}\right)$, obviously $u^{\prime}(1)<0$. 
Finally, we show $\left\|\overline{w u^{\prime}}\right\|_{\infty}<N$ for some $N>0$. Since $0 \leq u(t) \leq u_{*}(t)$ for all $t \in[0,1]$, by the similar calculation in the proof of Lemma 5.6, we have

$$
\left|\overline{w u^{\prime}}(t)\right| \leq 2^{(2-p) /(p-1)}\left[\left|Y^{\prime}(1)\right|+\left(2^{(p-2) /(p-1)}+1\right) a+\varphi_{p}^{-1}\left(f_{*} \max _{t \in[0,1]}\left\{w(t)^{p-1} \int_{t}^{1} s^{-\alpha} d s\right\}\right)\right] \triangleq N
$$

for all $t \in[0,1]$, where $f_{*}=\lambda \max _{u \in\left[0,\left\|u_{*}\right\|+a\right]} f(u)>0$, and

$$
Y(t)= \begin{cases}\int_{0}^{t} \varphi_{p}^{-1}\left(f_{*} \int_{s}^{B} \tau^{-\alpha} d \tau-\varphi_{p}(a)\right) d s+a t, & 0 \leq t \leq B, \\ \int_{t}^{1} \varphi_{p}^{-1}\left(f_{*} \int_{B}^{s} \tau^{-\alpha} d \tau+\varphi_{p}(a)\right) d s-a(1-t), & B \leq t \leq 1,\end{cases}
$$

and $B$ is defined by

$$
\int_{0}^{B} \varphi_{p}^{-1}\left(f_{*} \int_{s}^{B} \tau^{-\alpha} d \tau-\varphi_{p}(a)\right) d s+a B=\int_{B}^{1} \varphi_{p}^{-1}\left(f_{*} \int_{B}^{s} \tau^{-\alpha} d \tau+\varphi_{p}(a)\right) d s-a(1-B),
$$

and this completes the proof.

We now prove the main theorem in this section.

Proof of Theorem 5.1. Let $\lambda^{*}=\sup \left\{\mu \mid\left(\widehat{E}_{\lambda}\right)\right.$ have at least two positive solutions for all $\left.\lambda \in(0, \mu)\right\}$. Then, by Lemmas 5.5 and 5.6, $0<\lambda^{*} \leq \bar{\lambda}$. By the choice of $\lambda^{*},\left(\widehat{E}_{\lambda}\right)$ has at least two positive solutions for $\lambda \in\left(0, \lambda^{*}\right)$ and at least one positive solution at $\lambda=\lambda^{*}$. We will show that $\left(\widehat{E}_{\lambda}\right)$ has no positive solution for all $\lambda>\lambda^{*}$. On the contrary, assume that there exists $\lambda_{*}>\lambda^{*}$ such that $\left(\widehat{E}_{\lambda_{*}}\right)$ has a positive solution. We claim that $\left(\widehat{E}_{\lambda}\right)$ has at least two positive solutions for $\lambda \in$ $\left(\lambda^{*}, \lambda_{*}\right)$. Then, this contradicts to the definition of $\lambda^{*}$ and the proof is done. Define $M: K \rightarrow K$ by

$$
M u(t) \triangleq \begin{cases}\int_{0}^{t} \varphi_{p}^{-1}\left(\int_{s}^{A_{u}} \lambda \tau^{-\alpha} f(\gamma(u(\tau))+a(1-\tau)) d \tau-\varphi_{p}(a)\right) d s+a t, & 0 \leq t \leq A_{u}, \\ \int_{t}^{1} \varphi_{p}^{-1}\left(\int_{A_{u}}^{s} \lambda \tau^{-\alpha} f(\gamma(u(\tau))+a(1-\tau)) d \tau+\varphi_{p}(a)\right) d s-a(1-t), & A_{u} \leq t \leq 1,\end{cases}
$$

where

$$
\begin{aligned}
& \int_{0}^{A_{u}} \varphi_{p}^{-1}\left(\int_{s}^{A_{u}} \lambda \tau^{-\alpha} f(\gamma(u(\tau))+a(1-\tau)) d \tau-\varphi_{p}(a)\right) d s+a A_{u} \\
& \quad=\int_{A_{u}}^{1} \varphi_{p}^{-1}\left(\int_{A_{u}}^{s} \lambda \tau^{-\alpha} f(\gamma(u(\tau))+a(1-\tau)) d \tau+\varphi_{p}(a)\right) d s-a\left(1-A_{u}\right) .
\end{aligned}
$$


Then, $M: K \rightarrow K$ is completely continuous and $u$ is a solution of $\left(M_{\curlywedge 1}\right)$ if and only if $u=M u$ on $K$. By simple calculation, we can easily check that there exists $R_{1}>0$ such that $\|M u\|_{w}<R_{1}$, for all $u \in K$. Taking $R_{1}$ big enough satisfying $B_{R_{1}} \supset \Omega_{N}$ and applying Theorem 2.4 , we get

$$
i\left(M, B_{R_{1}} \cap K, K\right)=1 .
$$

By Lemma 5.7 and the excision property, we get

$$
i\left(M, \Omega_{N} \cap K, K\right)=i\left(M, B_{R_{1}} \cap K, K\right)=1 .
$$

Since problem $\left(\widehat{E}_{\mathcal{\lambda}}\right)$ is equivalent to problem $\left(M_{\lambda 1}\right)$ on $\Omega_{N} \cap K$, we conclude that $\left(\widehat{E}_{\mathcal{\lambda}}\right)$ has a positive solution in $\Omega_{N} \cap K$. Assume that $H(\lambda, \cdot)$ has no fixed point in $\partial \Omega_{N} \cap K$ (otherwise, the proof is done!). Then, $i\left(H(\lambda, \cdot), \Omega_{N} \cap K, K\right)$ is well defined and by (5.41), we have

$$
i\left(H(\lambda, \cdot), \Omega_{N} \cap K, K\right)=1 .
$$

By Lemma 5.5, we may choose $\lambda_{N_{0}}>\bar{\lambda}$ such that $\left(\widehat{E}_{\lambda_{N_{0}}}\right)$ has no solution in K. By a priori estimate (Lemma 5.6) with $I=\left[\lambda, \lambda_{N_{0}}\right]$, there exists $R_{2}>R_{1}$ such that for all possible positive solutions $u$ of $\left(\widehat{E}_{\mu}\right)$ with $\mu \in\left[\lambda, \lambda_{N_{0}}\right]$, we have

$$
\|u\|_{w}<R_{2}
$$

Define $h:[0,1] \times\left(\bar{B}_{R_{2}} \cap K\right) \rightarrow K$ by

$$
h(\tau, u)=H\left(\tau \lambda_{N_{0}}+(1-\tau) \lambda, u\right) .
$$

Then, by the similar argument as Lemma $5.4, h$ is completely continuous on $[0,1] \times K$ and by Lemma $5.6, h(\tau, u) \neq u$, for all $(\tau, u) \in[0,1] \times\left(\partial B_{R_{2}} \cap K\right)$. By the property of homotopy invariance, we have

$$
i\left(H(\lambda, \cdot), B_{R_{2}} \cap K, K\right)=i\left(H\left(\lambda_{N_{0}} \cdot\right), B_{R_{2}} \cap K, K\right)=0
$$

By the additive property and (5.42), we have

$$
i\left(H(\lambda, \cdot),\left(B_{R_{2}} \backslash \bar{\Omega}_{N}\right) \cap K, K\right)=-1
$$

Therefore $\left(\widehat{E}_{\lambda}\right)$ has another positive solution in $\left(B_{R_{2}} \backslash \bar{\Omega}_{N}\right) \cap K$. This completes the claim.

Corollary 5.8. Assume that $l>p$ and $q>p-1$. Then, there exists $\mu^{*}>0$ such that $(E)+\left(D_{1}\right)$ has at least two positive radial solutions for $\mu \in\left(0, \mu^{*}\right)$, at least one positive radial solution for $\mu=\mu^{*}$, and no positive radial solution for $\mu \in\left(\mu^{*}, \infty\right)$. 


\section{Nonexistence}

In this section, we will prove the nonexistence result of nonnegative solution of problem $\left(E_{\lambda}\right)$ if $\alpha \geq p$.

Theorem 6.1. Assume that $\left(F_{1}\right)$ holds and also assume $\alpha \geq p$. Then, $\left(E_{\curlywedge}\right)$ has no positive solution.

Proof. First, we prove the case $\alpha=p$. It is obvious that problem $\left(E_{\lambda}\right)$ does not have a trivial solution. Suppose on the contrary that there is a positive solution $u$ of $\left(E_{\lambda}\right)$ when $\alpha=p$. Since $u$ is concave, there exists unique $z \in(0,1)$ such that $u(z)=a / 2$. Therefore, we have $u(t) \geq a / 2$, for $t \in(0, z)$. This implies

$$
-\varphi_{p}\left(u^{\prime}\right)^{\prime} \geq \lambda m t^{-p}
$$

for $t \in(0, z)$, where $m=\min _{v \in[a / 2,\|u\|]} f(v)>0$. For $x \in(0, z / 2)$, we can easily know that

$$
x^{1-\alpha}-z^{1-\alpha}>\left(1-2^{1-\alpha}\right) x^{1-\alpha} .
$$

Integrating (6.1) from $x$ to $z$ and by (6.2) we obtain

$$
\varphi_{p}^{-1}\left[\varphi_{p}\left(u^{\prime}(x)\right)-\varphi_{p}\left(u^{\prime}(z)\right)\right] \geq C x^{-1},
$$

where $C=\varphi_{p}^{-1}\left(\lambda m(1 /(p-1))\left(1-2^{1-p}\right)\right)$. Since $\varphi_{p}\left(u^{\prime}(x)\right)-\varphi_{p}\left(u^{\prime}(z)\right) \geq 0$, we obtain

$$
x^{-1} \leq \gamma_{1 /(p-1)} C^{-1}\left[\left|u^{\prime}(x)\right|+\left|u^{\prime}(z)\right|\right],
$$

where $\gamma_{q}=\max \left\{1,2^{q-1}\right\}$. It is enough to consider the following two cases: (i) there exists $l \in$ $(0, z / 2)$ such that $u^{\prime}(x)>0$, for all $x \in(0, l)$ and (ii) $u^{\prime}(x)<0$ for all $x \in(0, z / 2)$. For the first case, let $y \in(0, l)$, then integrating (6.4) from $y$ to $l$, we get

$$
u(y) \leq u(l)+\left|u^{\prime}(z)\right|(l-y)+r_{1 /(p-1)}^{-1} C(-\ln l+\ln y) .
$$

Letting $y \rightarrow 0$, we have $u(y) \rightarrow-\infty$ and this is a contradiction to $u(0)=a$. For the second case, let $y \in(0, z / 2)$, then integrating (6.4) from $y$ to $z / 2$,

$$
\ln \frac{z}{2}-\ln y \leq \gamma_{1 /(p-1)} C^{-1}\left[-u\left(\frac{z}{2}\right)+u(y)+\left|u^{\prime}(z)\right|\left(\frac{z}{2}-y\right)\right] .
$$

This implies

$$
u(y) \geq u\left(\frac{z}{2}\right)-\left|u^{\prime}(z)\right|\left(\frac{z}{2}-y\right)+\gamma_{1 /(p-1)}^{-1} C\left(\ln \frac{z}{2}-\ln y\right) .
$$

Letting $y \rightarrow 0$, we obtain $u(y) \rightarrow \infty$. Once again, this is a contradiction to $u(0)=a$. Similarly, we can prove the same conclusion for the case $\alpha>p$.

Remark 6.2. Instead of condition $\left(F_{1}\right)$, if we assume that there exists $\epsilon>0$ such that $f(u)>0$ for $u \geq \epsilon$, then the conclusion of Theorem 6.1 still remains true.

Corollary 6.3. Assume $l \leq p$. Then $(E)+\left(D_{1}\right)$ has no positive radial solution. $[2]$.

One of the referee informed that Corollary 6.3 was proven under more general set up in 


\section{Acknowledgments}

The authors express their thanks to the referees for their valuable comments and suggestions. This work was supported by the Korea Research Foundation Grant funded by the Korean Government (MOEHRD)(KRF-2005-070-C00010).

\section{References}

[1] Y. Deng and Y. Li, "On the existence of multiple positive solutions for a semilinear problem in exterior domains," Journal of Differential Equations, vol. 181, no. 1, pp. 197-229, 2002.

[2] T. Kusano, A. Ogata, and H. Usami, "Oscillation theory for a class of second order quasilinear ordinary differential equations with application to partial differential equations," Japanese Journal of Mathematics. New Series, vol. 19, no. 1, pp. 131-147, 1993.

[3] Y. Naito and H. Usami, "Oscillation criteria for quasilinear elliptic equations," Nonlinear Analysis: Theory, Methods \& Applications, vol. 46, no. 5, pp. 629-652, 2001.

[4] E. Zeidler, Nonlinear Functional Analysis and Its Applications. I, Springer, New York, NY, USA, 1985.

[5] T Kusano, J. Jaroš, and N. Yoshida, "A Picone-type identity and Sturmian comparison and oscillation theorems for a class of half-linear partial differential equations of second order," Nonlinear Analysis: Theory, Methods \& Applications, vol. 40, no. 1-8, pp. 381-395, 2000.

[6] D. J. Guo and V. Lakshmikantham, Nonlinear Problems in Abstract Cones, vol. 5 of Notes and Reports in Mathematics in Science and Engineering, Academic Press, Boston, Mass, USA, 1988.

[7] R. P. Agarwal, H. Lü, and D. O'Regan, "Eigenvalues and the one-dimensional $p$-Laplacian," Journal of Mathematical Analysis and Applications, vol. 266, no. 2, pp. 383-400, 2002.

[8] M. del Pino, M. Elgueta, and R. Manásevich, "A homotopic deformation along $p$ of a Leray-Schauder degree result and existence for $\left(\left|u^{\prime}\right|^{p-2} u^{\prime}\right)^{\prime}+f(t, u)=0, u(0)=u(T)=0, p>1$," Journal of Differential Equations, vol. 80, no. 1, pp. 1-13, 1989.

[9] M. Zhang, "Nonuniform nonresonance of semilinear differential equations," Journal of Differential Equations, vol. 166, no. 1, pp. 33-50, 2000.

[10] C. Kim and Y. H. Lee, "Existence of multiple positive solutions for a $p$-Laplacian problem with $L^{1}$ indefinite weight," preprint. 